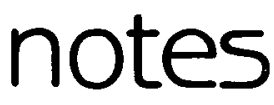

\title{
Le travail en présence de rayonnements ionisants dans des laboratoires universitaires
}

\author{
C. DEMERS, N. VÉZINA, K. MESSING*
}

(Manuscrit recu le 15 mai 1990)

RÉSUMÉ L'accroissement de l'utilisation des produits radioactifs dans les laboratoires de recherche a généré de nouveaux risques pour les travailleurs de laboratoires. En milieu universitaire, l'arrivée régulière de nouveaux étudiants pose des problèmes particuliers. A l'aide de méthodes d'analyse ergonomiques, nous avons étudié les conditions d'utilisations des produits radioactifs dans quatre laboratoires de deux universités québecoises. Nous avons observé deux travailleurs ou plus par laboratoire durant 34 heures de manipulations de produits radioactifs. Nous avons également mesuré le niveau de contamination par la technique de frottis dans tous les laboratoires. Les règles et pratiques de travail varient selon le laboratoire de recherche et selon l'expérience de l'individu. Les sites contaminés étaient surtout les espaces communs et les sites occupés par de nouveaux utilisateurs. Le port du dosimètre n'était pas suffisamment constant pour assurer que la dose se mesure avec précision.

Nos résultats nous permettent de classer les conditions pouvant comporter un risque d'exposition en trois catégories : 1) celles relevant de l'équipement, par exemple des écrans de dimensions insuffisantes ; 2) celles relevant des installations, par exemple un seul laboratoire maintenant un espace réservé à la manipulation des produits radioactifs ; 3 ) celles relevant de la formation du personnel en vue d'empêcher une contamination accrue des travailleurs ou des espaces avoisinants.

ABSTRACT The increasing use of radioactive material in research laboratories has generated new risks for laboratory workers. In universities, new students arrive constantly, posing a specific problem for radiation-protection. Using methods derived from ergonomic studies, we analysed the conditions of use of radioactive substances in four different laboratories - molecular biology (2), chemistry (1) and geology (1) in two universities in Quebec. We observed two or more workers in each of the laboratories during a total of 34 hours of handling of radioactive substances. We also measured radioactive contamination by surface sampling techniques in all laboratories. Work practices and regulations varied among laboratories and according to workers' experience. Contaminated sites were principally those in common use or those assigned to new students. Dosimeters were not worn with sufficient regularity for us to be confident that radiation doses were being accurately measured.

Our results permitted us to classify conditions at risk for exposure to radiation in three categories : 1) those associated with equipment, e.g., insufficient size of protective screen ; 2) those associated with laboratory installations, e.g., only one laboratory maintained a specific area for the handling of radioactive substances ; 3 ) those associated with training of personnel, resulting in use of techniques to control contamination of the worker or surroundings.

* Groupe de recherche-action en biologie du travail, CINBIOSE, Université du Québec à Montréal, Département des sciences biologiques, C.P. 8888, Succ. A, Montréal, Canada, H3C 3P8. 
L'utilisation de sources de rayonnements ionisants augmente dans les laboratoires universitaires en raison de l'introduction de nouvelles techniques employant des substances radioactives. Cet accroissement ne s'est pas fait sans susciter certaines inquiétudes puisqu'actuellement on estime qu'il n'y a pas de seuil d'exposition aux rayonnements ionisants sans effet génétique (Denniston, 1982, Upton, 1987).

Les conditions d'expositions aux rayonnements dans les laboratoires universitaires diffèrent de celles qui prévalent dans l'industrie ou l'hôpital. Dans les universités, le personnel de recherche peut se composer d'étudiants plus ou moins qualifiés en manipulation expérimentale. Le fait que les postes de techniciens de recherche dans les universités canadiennes dépendent, dans la plupart des cas, de subventions ponctuelles, entraîne de plus un certain roulement chez le personnel salarié. Les universités partagent avec d'autres instances le problème que les locaux dans lesquels on utilise les produits radioactifs n'ont pas nécessairement été conçus en prévision de cet usage et qu'il est difficile d'en limiter l'accès aux seuls usagers.

II existe plusieurs études sur l'exposition aux rayonnements dans le milieu universitaire. Pomroy a mesuré des contaminations internes supérieures à $9 \mathrm{nCi}$ à l'aide d'un compteur à scintillation placé à proximité de la thyroïde chez 17 des 52 personnes qui travaillaient avec l'iode 125 dans des laboratoires de quatre universités canadiennes, dont plusieurs n'avaient que peu de connaissance des méthodes de radioprotection [6]. A l'Université Sussex, en Angleterre, des chercheurs ont recommandé certaines méthodes de radioprotection suite à la mesure, chez un travailleur exposé au phosphore 32, d'une dose équivalente à $26 \mathrm{mSv} / \mathrm{mois}$ [1]. Une étude française a permis d'estimer que près de la moitié des 2740 personnes œuvrant dans des laboratoires était exposée à un risque par un agent physique, dont le quart par les rayonnements ionisants [5].

Ces recherches se limitaient à l'étude des mesures d'exposition ou à l'efficacité des méthodes de radioprotection. Elles nous ont incités à vérifier les conditions d'utilisation des sources de rayonnements ionisants dans des laboratoires québécois de recherche universitaire. Cette étude, en contraste avec celles citées ci-dessus, ne visait pas à estimer le degré de risque subi par les utilisateurs, mais plutôt à examiner l'activité de travail en vue d'identifier des situations susceptibles de provoquer une exposition accidentelle aux rayonnements.

\section{REETMODOLOGIE}

Nous avons observé les conditions d'utilisation des produits radioactifs et les niveaux de contamination pendant quatre mois durant l'été 1987 dans quatre laboratoires. L'étude a été faite en utilisant des méthodes propres aux recherches ergonomiques, dont l'objectif est l'analyse de l'activité de travail. Les méthodes utilisées 
comprennent des observations préliminaires, des observations systématiques et l'enregistrement des activités gestuelles, des postures, des incidents et des irrégularités de l'activité. Nous avons également tenté de cerner les contraintes environnementales et temporelles du travail [4].

Nous avons examiné les moyens mis en œuvre par les utilisateurs pour se protéger des expositions aux rayonnements ionisants: 1) les aménagements, 2) le blindage, 3) la distance, 4) le temps d'exposition et 5) les pratiques de travail. Finalement, nous avons fait certaines vérifications du niveau de la contamination dans les laboratoires où l'on manipule les substances radioactives. Ces données servaient à identifier des situations à risque de contamination et d'accidents.

\section{Inventaire des sources de rayonnements ionisants}

Dans le but de choisir des sites pour des observations détaillées, nous avons visité vingt laboratoires universitaires pour déterminer:

1. la source de rayonnement, par ex. phosphore 32 ;

2. la quantité utilisé, par ex. $25 \mathrm{mCi} / \mathrm{an}$;

3. les conditions de son utilisation, par ex. source scellée ou non, fréquence d'utilisation, risques de contamination, etc.

Cet inventaire nous fournissait les informations nécessaires pour sélectionner quatre laboratoires que nous voulions représentatifs des types d'utilisation de sources de rayonnements ionisants dans ces institutions. Les laboratoires ont été sélectionnés (tableau I) selon trois critères:

1. la manipulation de quantités appréciables de radioisotopes;

2. la possibilité d'observer plusieurs types de manipulations de radioisotopes ;

3. la diversité des sources de rayonnements ionisants.

TABLEAU ।

Description des laboratoires observés

\begin{tabular}{|c|c|c|c|c|c|}
\hline 1 & Chimie & $\begin{array}{l}{ }_{32}^{3} \mathrm{H} \\
32 \mathrm{P} \\
35 \mathrm{~S}\end{array}$ & $\begin{array}{c}\text { Source ouverte } \\
\text { liquide radio- } \\
\text { actif }\end{array}$ & $\begin{array}{l}1 \\
2 \\
2\end{array}$ & $\begin{array}{c}\text { Tech } \\
1^{e r} \text { cycle } \\
2^{e} \text { cycle }\end{array}$ \\
\hline 2 & $\begin{array}{c}\text { Biologie } \\
\text { moléculaire }\end{array}$ & ${ }^{32} \mathrm{P}$ & $\begin{array}{c}\text { Source ouverte } \\
\text { liquide radio- } \\
\text { actif }\end{array}$ & $\begin{array}{l}1 \\
1\end{array}$ & $\begin{array}{l}1^{\text {er }} \text { cycle } \\
2^{e} \text { cycle }\end{array}$ \\
\hline 3 & $\begin{array}{l}\text { Cristallo- } \\
\text { graphie }\end{array}$ & $\begin{array}{l}\text { Emetteur } \\
\text { de } \\
\text { rayons-X }\end{array}$ & Source fermée & $\begin{array}{l}1 \\
2\end{array}$ & $2^{e^{\text {Tech. }}}$ cycle \\
\hline 4 & $\begin{array}{c}\text { Biologie } \\
\text { moléculaire }\end{array}$ & ${ }^{32} \mathrm{p}$ & $\begin{array}{c}\text { Source ouverte } \\
\text { liquide radio- } \\
\text { actif }\end{array}$ & 2 & $2^{e}$. cycle \\
\hline
\end{tabular}




\section{Entrevues et observations}

1) Des entrevues ont été faites en vue de déterminer la dosimétrie employée, les aménagements des laboratoires, les règles de sécurité en usage concernant les sources de rayonnements ionisants et les pratiques de travail avec des radioisotopes.

2) Par la suite, nous avons observé des personnes à divers degrés d'expérience avec les différentes techniques. Cette première série d'observations qualitatives nous servait à identifier des situations où il existe un risque d'irradiation ou de contamination.

3) Une deuxième série d'observations a été faite pour apporter une dimension quantitative à la description de deux situations à risque. Ces observations ont duré $10 \mathrm{~h}$ s'échelonnant sur deux jours de travail consécutifs.

\section{Dépistage de contamination}

Dans tous les laboratoires où la source de rayonnements ionisants était un radioisotope, nous avons évalué la contamination par des frottis selon la méthode prescrite par la Commission de contrôle de l'énergie atomique (CCEA) du Canada.

\section{Méthode :}

A l'aide d'un papier-filtre humecté d'eau distillée, nous avons frotté une surface d'environ $100 \mathrm{~cm}^{2}$ ou, dans le cas d'objets tels que les pipettes, pinces et robinets, toute la surface de l'objet. Le papier-filtre était ensuite déposé dans une fiole à scintillation avec $10 \mathrm{ml}$ d'Aquasol et compté dans un compteur à scintillation. Un contrôle (papier-filtre $+10 \mathrm{ml}$ d'Aquasol) était inclu dans l'ensemble des échantillons.

\section{RÉSULTATS}

\section{Inventaire}

Lors de l'étude, 26 radioisotopes figuraient sur les permis de possession émis par la CCEA pour les départements de biologie, chimie et des sciences de la terre. De ce nombre, 6 radioisotopes étaient utilisés lors de notre inventaire : le tritium, le carbone 14 , le sodium 22, le phosphore 32 , le soufre 35 et l'iode 125 . En plus des radioisotopes, nous avons relevé la présence d'un appareil à diffraction émetteur de rayons $X$ moins énergétiques que ceux utilisés en radiologie médicale, c'est-à-dire inférieurs à $50 \mathrm{keV}$.

\section{Entrevues et observations}

Impossibilité de connaître avec précision la dose du personnel

La plupart du personnel n'a enregistré aucune dose pour la période d'observation, entre juin et septembre 1987. Mais ce résultat de dosimétrie ne témoigne pas nécessairement d'un faible niveau d'exposition. La 
plupart des utilisateurs de sources de rayonnements ionisants employaient des dosimètres thermoluminescents accrochés à la pochette de leur sarrau. L'emploi du dosimètre était donc relié au port du sarrau, qui n'est pas régulier. Une seule personne utilisait un dosimètre de type "bague" pour estimer l'exposition au niveau de ses membres supérieurs lors du travail derrière écran de protection (voir ci-dessous).

De plus, l'usage des dosimètres est réservé aux personnes qui travaillent directement avec des sources de rayonnements ionisants, excluant celles qui partagent les locaux avec elles. L'appareil à rayons $X$ se situe dans un local où nous avons observé la présence de deux personnes qui ne portaient pas de dosimètre. Ces personnes passaient souvent plus de temps en présence de l'appareil en marche que le technicien affecté à l'appareil (tableau II).

\section{TABLEAU ॥}

Temps passé par trois usagers du local où est installé l'appareil à diffraction (laboratoire 3):

(Période observée) : $10 \mathrm{~h}$ pendant que l'appareil à diffraction est en

1 : technicien, 2 et 3 : utilisateurs de l'ordinateur

\begin{tabular}{|lcccc|}
\hline Personne observée : & 1 & 2 & 3 \\
\hline \hline $\begin{array}{l}\text { Distance de la source de rayonnements } \\
\text { ionisants }(\mathrm{m})\end{array}$ & $<0,3^{\mathrm{a}}$ & $2,3^{\mathrm{b}}$ & $2,3^{\mathrm{b}}$ \\
\hline \hline $\begin{array}{l}\text { Temps passé dans le local: } \\
\text { (min) }\end{array}$ & 59 & 131 & 105 \\
\hline
\end{tabular}

a) porte un dosimètre (lecture $=0 \mathrm{mSv}$ pour les 3 mois sous étude) et quitte la pièce lorsque l'appareil est en marche.

b) demeurent dans la pièce lorsque l'appareil est en marche et ne portent pas de dosimètre.

\section{Conditions pouvant amener une exposition accidentelle : l'équipement}

Ecrans: Trois types d'écrans sont utilisés dans les deux institutions. Le premier type d'écran est utilisé pour des manipulations qui durent de 2 à $4 \mathrm{~h}$, nécessitant donc une position confortable. La personne doit passer ses bras au-dessus de l'écran pour réaliser sa manipulation, et doit donc s'approcher très près de l'écran pour ne pas être obligée de travailler les bras surélevés et étendus. Pour avoir une posture confortable de travail, le visage devait être au-dessus de l'écran, donc le cou et la tête de l'utilisateur n'étaient pas protégés par l'écran. Les expositions du cou, de la tête et des membres ne peuvent pas être enregistrées sur un dosimètre situé derrière l'écran. Le deuxième type d'écran est utilisé pour des manipulations brèves durant entre 5 et $20 \mathrm{~min}$. II est beaucoup plus haut et large, et permet de limiter l'exposition des parties périphériques du corps. Toutefois, les utilisateurs y travaillent en passant leurs bras de chaque côté de l'écran; ce qui rendrait difficile de longues manipulations. Le troisième type d'écran est plombé et est utilisé pour le travail sous hotte 
chimique avec des radioisotopes qui émettent des radiations pénétrantes. II s'agissait, dans le cas étudié, d'échantillons de minerai irradiés qui émettent des rayons gamma (la radioactivité émise par 10 échantillons pris au hasard $6 \mathrm{j}$ après l'irradiation fut de 100 à $150 \mathrm{rem} / \mathrm{h}$, soit 1 à $1,5 \mathrm{~Sv} / \mathrm{h}$ ). II est nécessaire de faire des compromis entre une hauteur adéquate de l'écran et les proportions de la hotte chimique.

Disposition des déchets: Dans un des laboratoires où nous avons fait des observations, nous avons remarqué que lors des manipulations des produits radioactifs, les utilisateurs jetaient du matériel susceptible d'être contaminé dans une poubelle ordinaire située sous leur table de travail, vidée à la fin de la manipulation dans un conteneur à déchets radioactifs. Ceci entraîne un risque de confusion pour les autres personnes. Dans le laboratoire 2, les utilisateurs avaient de la difficulté à vider la solution radioactive contenue dans un récipient en verre dans le bidon à déchets radioactifs liquides sans qu'il s'en échappe sur le sol, car ces conteneurs n'étaient pas conçus en fonction de cet usage. Dans deux autres laboratoires, nous avons constaté que les bidons à déchets liquides radioactifs restaient constamment ouverts même lorsqu'il n'y avait pas de manipulations de radioisotopes, ce qui représente un risque de contamination accidentelle.

\section{Conditions pouvant amener une exposition accidentelle: les aménagements}

Les espaces de rangement dans ces laboratoires étaient inadéquats. Dans un des laboratoires, nous avons observé que le conteneur à déchets radioactifs et le bidon à liquides radioactifs étaient placés à un endroit où ils pouvaient être renversés s'ils sont heurtés par les usagers de la hotte. Les usagers de ce laboratoire plaçaient leurs effets personnels sous la table où étaient réalisées les manipulations de sources radioactives. Dans un second laboratoire, beaucoup de matériel reposait sur les tables, un espace minimal étant dégagé pour travailler avec les radiations. Un seul laboratoire avait un espace spécifiquement consacré aux manipulations des radioisotopes. Cette solution était rejetée par d'autres laboratoires en raison d'une crainte de contamination résultant du partage des espaces.

Certains utilisateurs doivent se déplacer en portant des produits radioactifs, parfois à l'intérieur du laboratoire, parfois même d'un étage à un autre. Ainsi, le compteur à scintillation se situe au rez-de-chaussée alors que plusieurs utilisateurs travaillent au sous-sol. D'autres utilisateurs se servent d'un congélateur à très basse température situé dans un espace commun au sous-sol.

\section{Conditions pouvant amener une exposition accidentelle: les manipulations}

En l'absence de politique officielle concernant l'entraînement des nouveaux utilisateurs, il incombe à chaque chercheur individuel de former les usagers de son laboratoire. Les utilisateurs n'ont pas de techniques de travail uniformes. 
Les utilisateurs du laboratoire 1 doivent toucher à beaucoup d'objets tels que les pinces pour les filtres, les agitateurs Vortex, les boîtes contenant les embouts de pipettes, les fioles à scintillations, les robinets d'éviers et même le téléphone. Comme l'ensemble de ces manipulations sont faites alors que les utilisateurs portent des gants jetables éventuellement contaminés, nous avons évalué la fréquence des changements de gants. Nous avons choisi d'évaluer cette pratique qui varie beaucoup d'un individu à un autre. (tableau III).

TABLEAU III

Nombre de changements de gants pour trois personnes du laboratoire 1

Période d'observation: $2 \mathrm{~h} 15 \mathrm{~min}$.

Type de manipulation: Voir tableau I, laboratoire 1.

\begin{tabular}{|lllll|}
\hline Personne observée : & 1 & 2 & 3 \\
\hline Nombre de changements de gants & $2^{(a)}$ & 6 & $3^{(b)}$ \\
\hline
\end{tabular}

(a) : L'utilisateur a répondu au téléphone sans enlever ses gants.

(b) : Durant cette période, l'utilisateur a pris des solutions au réfrigérateur et a mis des échantillons dans le compteur à scintillation sans changer de gants avant de faire ces manipulations.

Au laboratoire 1, une manipulation consiste à vider le contenu radioactif d'une éprouvette sur un filtre installé sur un porte-filtre, à rincer l'éprouvette et à vider la solution de rinçage sur le filtre. A chaque fois, une certaine quantité de liquide coule par dessus le porte-filtre. Cette étape étant répétée jusqu'à 150 fois dans une journée, une quantité appréciable de liquide se retrouve sur le papier absorbant qui recouvre le comptoir.

II n'y a pas eu de manipulation de poudres pendant la période d'observation, mais nous avons noté que ces manipulations de mise en solution des poudres radioactives entraînent des éclaboussures car les fenêtres des hottes sont tachées. Cette observation a été confirmée par le technicien qui fait la vérification des contaminations radioactives.

\section{Frottis}

Nous avons effectué 63 frottis dans des laboratoires où l'on travaille avec des radioisotopes ainsi que dans les locaux où sont entreposés les déchets. La contamination est très variable d'un local à un autre et selon l'utilisateur individuel. Par exemple, la contamination des robinets à air à 4 postes de travail varie entre 582 et $19284 \mathrm{cpm}$, des surfaces de travail entre "indétectable" $(<100 \mathrm{cpm})$ et $1450 \mathrm{cpm}$, des poignées de diverses portes entre "indétectable" et $1202 \mathrm{cpm}$, et des planchers aux différents locaux entre "indétectable" et $5966 \mathrm{cpm}$. Un tiroir contenant des pipettes enregistrait $14509 \mathrm{cpm}$. Les niveaux les plus contaminés correspondaient soit à du personnel non-initié, soit à des espaces communs. Au laboratoire 4, par exemple, le site le plus contaminé est celui où travaillait l'utilisateur le moins expérimenté ; un 
niveau de contamination de $762 \mathrm{cpm}$ a été mesuré sur son comptoir alors que la contamination des autres est presque toujours indécelable et ne dépasse jamais $200 \mathrm{cpm}$. Dans les espaces communs, des frottis ont révélé des niveaux de contamination variant entre 382 et $5966 \mathrm{cpm}$ sur la poignée extérieure de la porte, sur le sol du local et sur l'interrupteur de lumière. Au laboratoire 1, le nombre de sites contaminés a quadruplé entre janvier et juillet 1987, passant de 3 à 12 sur 23 sites échantillonnés régulièrement. Cette augmentation correspond à la fois à l'arrivée de nouveaux étudiants au laboratoire en mai et à l'augmentation des manipulations des produits radioactifs par le personnel en place durant cette période.

\section{DISCUSSION ET CONCLUSIONS}

Nous ne pouvons pas estimer avec précision le degré de risque d'exposition du travail en laboratoire de recherche. De plus, nous ne pouvons pallier la difficulté que les pratiques liées à la volonté individuelle puissent être altérées en notre présence. Toutefois, nous avons pu constater un certain nombre d'aspects problématiques au niveau de la dosimétrie, des aménagements et des pratiques de travail.

La dosimétrie est faite de façon plutôt aléatoire : certaines personnes potentiellement exposées ne portent pas de dosimètre, même celles qui en ont les portent de façon sporadique, et les parties du corps les plus exposées ne portent pas toujours le dosimètre.

Les règles et pratiques de travail varient énormément et laissent penser que le risque d'exposition accidentelle n'est pas nul. Ces pratiques diffèrent selon le laboratoire, selon les choix de l'individu, et selon l'expérience. Les pratiques élémentaires comme le changement de gants ne font pas l'objet d'un consensus, d'où une grande variation dans le niveau de contamination révélé par les frottis. Cette contamination semble intimement liée à une inexpérience de manipulation et disparaît vraisemblablement avec le temps. Toutefois, en milieu universitaire, l'arrivée constante de nouveaux étudiants ne constitue pas une situation exceptionnelle mais fait partie des conditions du travail sous rayonnements.

La montée de contamination qui coïncide avec l'arrivée de nouveaux utilisateurs au laboratoire 1 et le degré élevé de contamination au site de travail de la personne la moins expérimentée du laboratoire 4 suggèrent la nécessité de programmes plus formels de formation. Une telle formation pourrait également limiter l'anxiété des utilisateurs, dont certains se demandent constamment s'ils se sont contaminés. lls se posent des questions aussi quant au choix de manipuler rapidement ou en faisant attention à la sécurité. La nécessité de ce dernier choix serait diminuée avec un meilleur aménagement des espaces en fonction des contraintes et des postures du travail. 
II est possible que des expositions accidentelles soient passées inaperçues en raison du manque de mesures de contrôle. Certaines situations observées comportent une certaine probabilité d'exposition accidentelle à dose élevée : renversement de déchets ou de solutions radioactives, contamination cutanée par contact avec de nombreuses surfaces non-nettoyées, respiration de poudres et vapeurs radioactives.

Le nettoyage et la vérification des espaces communs semble être négligés, peut-être en raison du fait que le mode de gestion de la radioprotection passe par les chercheurs individuels. Un programme concerté d'inspection des lieux de travail et du contrôle de la contamination remédierait à ce problème.

Nos observations ont également mis en évidence le peu d'importance accordé au confort des utilisateurs dans la conception des installations où sont manipulés les radioisotopes. Comme l'utilisation des produits radioactifs augmente sans arrêt, il serait important d'équiper les laboratoires de recherches d'installations permanentes et sécuritaires et qui prennent en compte le confort des usagers qui doivent les utiliser régulièrement et durant de longues périodes.

\section{REMERCIEMENTS}

Nous remercions les personnes qui nous ont permis d'observer leur travail et les chercheurs qui nous ont donné accès à leur laboratoire. Nous remercions également l'Institut de recherche en santé et en sécurité du travail du Québec pour une subvention dans le cadre de leur programme d'équipes associées.

\section{BIBLIOGRAPHIE}

[1] BALLANCE P., MORGAN J. et al. - The preparation of radioactively labelled DNA. Safety Practitioner, 1984, 1, 25-26.

[2] DENNISTON C. - Low level radiation and genetic risk estimation in man, Ann. Rev. Genetics, 1982, 16, 329-355.

[3] GHYS R. - Manuel de radiobiologie et de radioprotection à l'usage des radiobiologistes et des techniciens en radiologie. Paris: Maloine, 1971.

[4] LAVILLE A., TEIGER C. - Quelques réflexions sur les méthodes en ergonomie. In : Conception des espaces industriels et amélioration des conditions de travail, Montréal: Presses de l'Université de Montréal, 1982, 131-135.

[5] LIVET D., BECK N., ANDLAUER P. - Les risques dans les laboratoires publics de recherche. Rev. Conditions Trav., 1988, 37, 31-35.

[6] POMROY C. - Surveys of lab technicians for 1-125 thyroid burdens. Occup. Health Safety, 1979, 48 (5) 40-42.

[7] UPTON A.C. - Prevention of work-related injuries and diseases. Lessons from experience with ionizing radiation. Am. J. Ind. Med., 1987, 12, 291-309. 